\title{
The status of neutrino interaction theory and its impact on future neutrino oscillation experiments.
}

\author{
M. Kabirnezhad* \\ University of Oxford \\ E-mail: minoo.kabirnezhadephysics.ox.ac.uk
}

\begin{abstract}
Over the last decade, there has been enormous effort to measure neutrino interaction cross sections important to oscillation experiments. However, a number of results from modern experiments appear to be in tension with our model predictions. It also has been known that our models are outdated and with assumptions for simplicity, therefore, many efforts have been devoted to the quasielastic peak and dip region to improve them but very little attention has been given to the resonance and higher regions.

The MK model provides a full kinematic description of single pion production in the neutrinonucleon interactions, including resonant and nonresonant interactions in the helicity basis, in order to study the interference effect. The MK model shows better agreements with data compare to the exciting (RS) model in NEUT.

The effects of new (MK) model on T2K oscillation measurements shows bias on $\Delta_{23}^{2}$ and $\theta_{23}$. Although bias is small with respect to the uncertainty right now, clearly mis-modelling can produce biased oscillation parameters, and this will be a serious problem for next generation experiments.
\end{abstract}

Neutrino Oscillation Workshop (NOW2018)

9 - 16 September, 2018

Rosa Marina (Ostuni, Brindisi, Italy)

\footnotetext{
* Speaker.
} 


\section{Introduction}

Current and planned accelerator-based neutrino oscillation experiments must operate in the intermediate $(0.1-10 \mathrm{GeV})$ difficult energy range, where neutrinos interact with a single nucleon via different interactions while they overlap in the same range of energy(see Fig. ??). Besides these experiments use nuclear targets such as ${ }^{12} \mathrm{C},{ }^{16} \mathrm{O}$ or ${ }^{40} \mathrm{Ar}$, where a number of nuclear effects complicate the modelling problem further. In addition, particles produced in neutrino-nucleon interactions can re-interact before leaving the nucleus and can be absorbed, change their kinematics or even charge before being detected which is called FSI effects. Because of FSI, it is not possible to unambiguously separate interaction modes in an experiment, it is only possible to measure the post-FSI final state particle content.

Neutrino interaction models are required to accurately predict the number and topology of ob-



Figure 1: $v$ CC cross-section per nucleon.

served neutrino interactions and to estimate source of backgrounds. Neutrino interaction models are embedded in software called an "event generator". Two examples of currently using generators in neutrino experiment measurements are NEUT [?](primary generator for T2K, SK, secondary for others) and GENIE [?] (widely used by experiments).

In this paper, we introduce MK model which describes single pion production (SPP) in neutrinonucleon interaction. SPP channels make up the largest fraction of the inclusive neutrino-nucleus cross section in the intermediate range(see Fig. ??), a region covered by most accelerator-based neutrino beams. Foundation of neutrino-nucleus interaction models are neutrino-nucleon reaction processes like the one described by the MK model.

\section{The MK model}

Single pion production from a single nucleon occurs when the exchange boson has the requisite four-momentum to excite the target nucleon to a resonance state which promptly decays to 
produce a final-state pion (resonant interaction) or to create a pion at the interaction vertex which is called nonresonant interaction.

The Rein and Sehgal (RS) model [?] which is a default model for NEUT and GENIE describes only resonant interaction and does not include a reliable model for nonresonant processes and related interference terms. It is not a full kinematic model and the output of this model is $d \sigma / d W d Q^{2}$ where it does not include the pion angles. In the new (MK) model the full kinematic cross section is calculated and the output of the model is $d \sigma / d W d Q^{2} d \theta_{\pi} d \phi_{\pi}$.

interactions are defined by a set of Feynman diagrams as shown in Fig. ??. The pseudovector $N N \pi$ vertices are determined by the HNV model [?].

RS model is based on helicity amplitudes derived in a relativistic quark model. Therefore, the
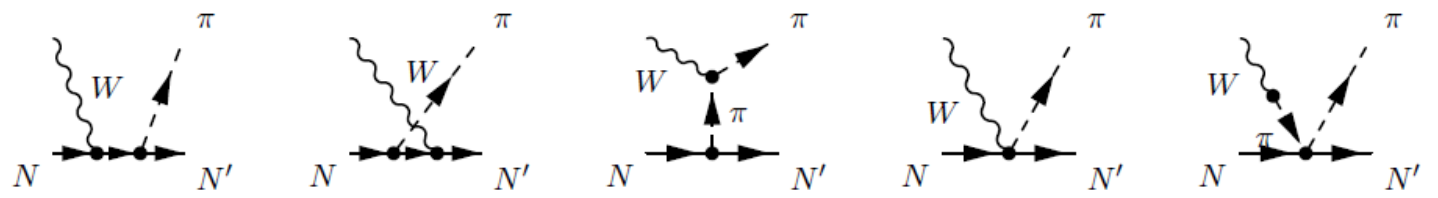

Figure 2: Nonresonant pion production diagrams from Ref. [?]

helicity amplitudes of the above diagrams should be calculated In order to add them to the resonant helicity amplitudes coherently.

\section{Results}

Implementing the MK model in NEUT enable us to do data comparison in any frame, in any physical region and any selection with any target. but we should keep in mind that MK model is only $1 \pi$ cross section model, therefore, we can only judge the model improvement with light targets such as hydrogen or deuterium. Fig ?? clearly shows that MK-model had a better agreement with data than the existing (RS) model in NEUT for pion momentum distribution in the lab frame. We
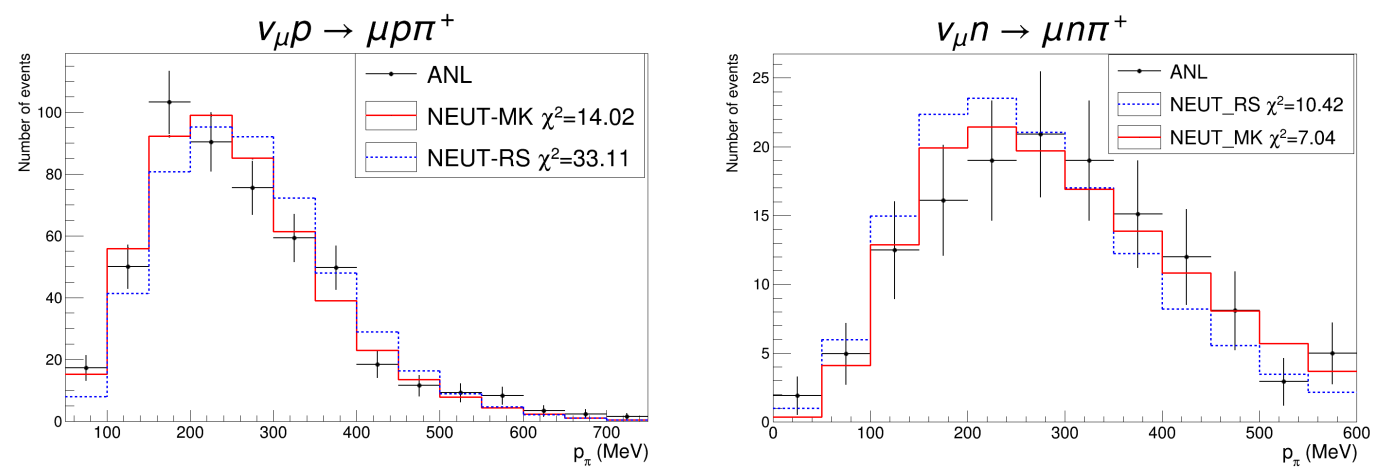

Figure 3: Pion momentum distribution for the $v p \rightarrow \mu p \pi^{+}$(left) and $v n \rightarrow \mu n \pi^{+}$(right) channels from ANL [?]. Model (solid red) and RS model (dashed blue) predictions of flux-averaged $p_{\pi}$-differential cross sections (with $W<2 \mathrm{GeV}$ cut), normalized to data are also presented for the two channels.

can also compare NEUT predictions (with RS and MK models ) with nuclear targets. Fig. ?? 
shows NEUT comparisons with Water and $\mathrm{CH}$ targets. As we mentioned before $1 \pi$ model is the only one piece that has been modified in this comparison for example the disagreement in right plot at low $Q^{2}$ for both NEUT predictions is due to nuclear effects.
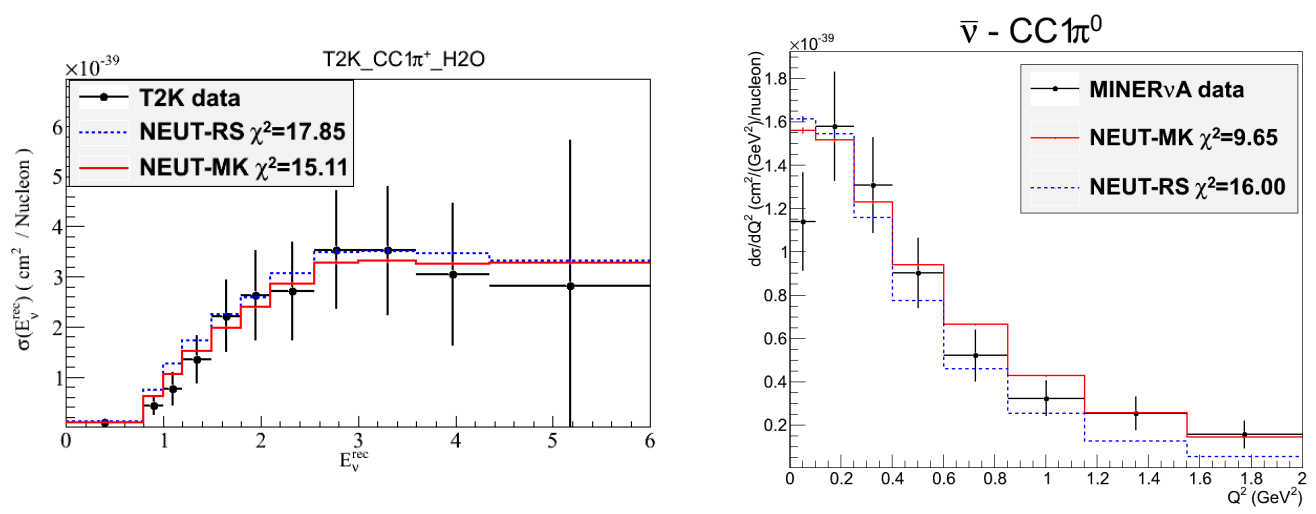

Figure 4: T2K measurements [?] on water for $\mathrm{CC} 1 \pi^{+}$differential cross-sections as a function of reconstructed neutrino energy (left) and MINERvA data [?] on $\mathrm{CH}$ target for $\bar{v} \mathrm{CC} 1 \pi^{0}$ differential cross-section in terms of $Q^{2}$ (right). Histograms show NEUT predictions with MK-model (solid-red) and RS-model (dashed-blue).

\section{Conclusion}

Current and future neutrino oscillation program critically depends on interaction models which are embedded in event generators such as NEUT. Precise neutrino oscillation measurements need precise neutrino interaction models. In this work, we developed a new $1 \pi$ cross section (MK) model that improves the existing (RS) model in NEUT. The MK model provides a differential cross section, $d \sigma=d W d Q^{2} d \omega$, for single pion production up to $\mathrm{W}=2$ $\mathrm{GeV}$. It consists of resonant and nonresonant interactions and includes interference effects. These model developments should be done for all models in event generators in order to get precise neutrino oscillation measurements.

\section{References}

[1] M. Kabirnezhad, Phys. Rev. D 97 (2018) no.1, 013002

[2] D. Rein, Z. Phys. C 35 (1987) 43.

[3] D. Rein and L. M. Sehgal, Annals Phys. 133 (1981) 79.

[4] E. Hernandez, J. Nieves and M. Valverde, Phys. Rev. D 76 (2007) 033005

[5] Y. Hayato, Acta Phys. Polon. B 40 (2009) 2477.

[6] C. Andreopoulos et al., Nucl. Instrum. Meth. A 614 (2010) 87

[7] M. Derrick et al., Phys. Rev. D 23 (1981) 569.

[8] K. Abe et al. [T2K Collaboration], Phys. Rev. D 95 (2017) no.1, 012010

[9] C. L. McGivern et al. [MINERvA Collaboration], Phys. Rev. D 94 (2016) no.5, 052005 